staccato sound. This point, taken as systolic blood pressure, is slightly lower than that by direct measurement, but is acceptably close, and there are no other sound criteria to confuse the issue.

As the extramural pressure is lowered further the proportion of the cardiac cycle during which the artery is patent progressively increases, causing the decrease in pitch and increase in length of the sound as it approaches diastolic pressure, but as long as this extramural pressure is above diastolic there will be a period, however brief, when the artery is closed and the sound will be staccato. As soon as the sound appears muffled this criterion no longer holds and this must be the closest approximation to the diastolic pressure, and direct measurement confirms this.

The disappearance of sound is in many cases close to the muffled point and can give a reasonable approximation of the diastolic blood pressure, but it is clearly illogical to suggest a correspondence of the two, for the following reasons. Firstly, in a hyperkinetic circulation, as the authors point out (for example, after exercise or the apprehensive patient in the examining room), sounds may be heard over the brachial artery even when the cuff pressure reads zero. Secondly, if the stethoscope bell is not at the point of maximum pickup over the artery, or in a fat arm, the sound will disappear at higher pressures and the same occurs if the stethoscope transmission is poor or the examiner's hearing impaired.

The continued recommendation of phase 5 as the diastolic pressure is, in my opinion, a grosser error than that of the almost universal practice of starting a pulse count on the unit as opposed to zero, thus negating the basic principle of interval timing.

\section{A MERCURIUS-TAYLOR}

Whittington Hospital,

London N19

\section{Neurological manifestations and} Mycoplasma pneumoniae infection

SIR,-I would like to add a further case report to those given by Drs J Aidan Twomey and M L E Espir in their short report "Neurological manifestations and Mycoplasma pneumoniae infection" (6 October, p 832).

The patient was a 54 -year-old woman who presented to the department of neurosurgery with a five-day history of atypical pneumonia and gradually progressive focal neurological signs suggestive of a left temporal lesion with a dense right hemiparesis. Investigations revealed a white blood count of $22.5 \times 10^{9} / 1$ (87\% neutrophils, $7 \%$ monocytes) associated with strongly positive cold agglutinins and a mycoplasma titre rising to $1 / 512$. The patient had been started on antibiotics prior to admission and mycoplasma was not cultured from blood, sputum, or cerebrospinal fluid. Analysis of the cerebrospinal fluid showed normal protein glucose, and cell counts and CSF IgG and IgA within the normal range. Computed tomography (CT) confirmed a large left temporal lesion of intermediate uptake but the brain biopsy specimen was histologically normal. The patient responded well to erythromycin, with complete recovery at her six months' follow-up, and a later CT scan showed resolution of the abnormal area.

I report the case as a neurological manifestation of mycoplasma pneumonia presenting as a focal lesion resembling an acute glioma. I suggest that the underlying pathology is direct infection of the nervous system rather than a secondary immunological reaction. While agreeing with the authors that mycoplasma involvement should be excluded in otherwise unexplained acute neurological symptoms I do not agree that its incidence is underestimated.

I am grateful to Mr K Frazer of the department of neurosurgery, University Hospital of Wales, Cardiff, for permission to report this case.

D B JONES

University Hospital of Wales,

Cardiff CF4 4XW

\section{Mycosis fungoides-unsolved problems}

SIR,-Your leading article "mycosis fungoides -unsolved problems" (18 August, p 409) is an excellent review of the diagnostic and therapeutic problems of this intricate disorder. We would, however, dispute the conclusion: "Which of all of these treatments is used must depend partly on the individual case, but even more on the techniques and experience available in any one centre."

We feel that this attitude suggests a lack of collaboration and exchange of information between centres, which contrasts sharply with the activities of those involved in research in mycosis fungoides. The establishment of cooperative study groups in Scandinavia, the Netherlands, Scotland, and North America is a result of this interest. The international meetings on mycosis fungoides in Paris in 1977 and Washington in 1978 formed valuable platforms for the dissemination of new knowledge between these study groups and emphasised the value of our continuing collaboration in increasing knowledge and understanding of this rare disease.

\section{Linköping} Ove Groth
LARS MOLIN Copenhagen

KRISTIAN THOMSEN

GUNNAR VOLDEN Scandinavian Mycosis Fungoides Study Group Oslo Rona MacKie

Scottish Mycosis Fungoides Group

Glasgow WILLEM VAN VLOTEN

Dutch Mycosis Fungoides Group Leiden

STANFORD LAMBERG

American Mycosis Fungoides

Co-operative Group Baltimore

Possible cancer hazard associated with

5-methoxypsoralen in suntan preparations

SIR,-In 1976 it was suggested ${ }^{1}$ that some degree of caution should be exercised in the use of psoralens in the phototherapy of psoriasis "as somatic chromosome damage of this magnitude may result, after clonal selection, in later malignancy." Stern $e t$ al has reported that the risk of cutaneous carcinoma in patients treated with oral 8-methoxypsoralen phototherapy for psoriasis was 2.63 times higher than expected for a matched control group. An accompanying editorial ${ }^{3}$ comments on the risks and benefits of phototherapy.

It is with some disquiet that I would inform your readers of what may be a serious problem to a very large number of young people using certain suntan preparations widely advertised and sold by pharmacies, without prescription, in several European countries, including France. French men and women as well as tourists flocking to the warm beaches and to the ski resorts are, in my judgment, at risk. The suntan preparations sold contain, in many formulations, 5-methoxypsoralen as well as the usual ultraviolet chemical filters. This furocoumarin has, as one of its effects, the ability to induce tanning in the presence of light (320-380 nm). This is, of course, the major desired effect of 5-methoxypsoralen and thus such preparations will help tanning. The melanin pigments will thus decrease the harmful effects of prolonged exposure to solar radiation.

The other biological effects of 5-methoxypsoralen, however, are similar to those of psoralen (the parent chemical) and 8-methoxypsoralen. There is an abundance of data $^{4}$ that psoralens, although differing quantitatively in their biochemical and biological effects, (a) form monoadducts and cross-links to DNA in the presence of light; (b) produce point mutations or chromosomal mutations (or both) in all cellular systems treated, from viruses to human cells; $(c)$ produce skin cancer, in the presence of light, in mice; and $(d)$ produce skin cancer (and possibly other cancers) in the presence of light in man. There is admittedly no direct evidence as yet that 5-methoxypsoralen is carcinogenic or mutagenic. There is nothing, however, in the chemical structure to indicate that it will behave in a qualitatively different manner from the other psoralens. Its reactions with DNA are discussed by Scott et al. ${ }^{4}$

It is with considerable uneasiness that I suspect an increase in skin and other cancers to be probable in future years from the widespread and, in my opinion, ill-advised use of preparations containing 5-methoxypsoralen. My concern is not helped by the knowledge that DNA repair mechanisms may be less than optimal in, perhaps, one person in 100 .

\section{J AsHWOOD-SMITH} University Department of Biology,
Victoria, V8W 2Y2, British Columbia, Canada

${ }^{1}$ Ashwood-Smith, M J, and Grant, E, British Medical Fournal, 1976, 1, 342.
Stern, R , et al, New England fournal of Medicine, 1979, 300, 809.

3 Epstein, J H, New England Fournal of Medicine, 1979, $300,852$. cott, B R, Pathak, M A, and Mohn, G R, Mutation
Research, 1976, 39, 29-74.

\section{Clinical practice and community} medicine

SIR,-Your leading article (6 October, p 817) and Professor E D Acheson's article (p 880) on this subject raise issues which have quite often been discussed in the past. There appears to be nothing in principle against the idea of a community physician having a part-time contract with his employing authority, the remainder of his time being spent in a subconsultant capacity in a clinical specialty. Many-community physicians, though they may hanker after clinical work, find that their regular employment is sufficiently demanding. There may be occasions when an employing authority, in order to fill a vacancy, will be glad to agree to such an arrangement. There are, however, practical and formal difficulties to be overcome in order to provide work which is suited to a particular individual's skills and interests. For example; there may 